\title{
Comparison of rapid immuno- chromatographic card test with elisa in diagnosis of dengue fever at tertiary care centre
}

\author{
Sarah Hassan ${ }^{1}$, Vineeta Khare ${ }^{2, *}$, Mastan Singh ${ }^{3}$, Syed Abid Asghar ${ }^{4}$ \\ ${ }^{1,4}$ Junior Resident, ${ }^{2}$ Professor, ${ }^{3}$ Professor \& HOD, ${ }^{1,2,3}$ Dept. of Microbiology, ${ }^{4}$ Dept. of Community Medicine, Era's Lucknow \\ Medical College and Hospital, Lucknow, Uttar Pradesh, India
}

*Corresponding Author:

Email: vinitakhare@yahoo.com

\begin{abstract}
Introduction: Dengue viruses are Arboviruses capable of infecting humans, and causing diseases ${ }^{1}$.Dengue virus belongs to the family Flaviviridae, genus Flavivirus. The Aedes aegypti mosquito is the principal vector that transmits the viruses that cause dengue. The viruses are passed on to humans through the bites of an infective female Aedes mosquito, which acquires the virus while feeding on the blood of an infected individual. Commercially available rapid kits, detecting the presence of NS1 antigen and anti-dengue antibodies have been developed for the rapid detection of dengue.Diagnosis of dengue at an early stage is the only effective way to control disease progression.

Objective: To compare rapid immunochromatographic test with ELISA for detection of NS1antigen and Ig M in suspected dengue patients.

Materials and Methods: A Cross-Sectional study was carried out in the Department of Microbiology, Era's Lucknow Medical College and Hospital from the month of July 2016 to September 2016.Serum samples of 225 suspected dengue patients were collected, having fever for 5 days or less. All samples were analyzed by Dengue Day 1 Test which detects NS1 antigen, IgM and IgG antibodies; by Panbio Dengue Early ELISA for detecting NS1 antigens and by Panbio Dengue IgM Capture ELISA for IgM antibodies.

Results: The Rapid Dengue Day 1 card Test showed a sensitivity and specificity of $92 \%$ and $98 \%$ for NS1 antigen detection and $90 \%$ and $100 \%$ for IgM Antibody detection.

Conclusion: High sensitivity and specificity of rapid diagnostic tests for early detection of dengue was observed. These kits are highly suitable for early detection of positive cases, as with high sensitivity and specificity it can help in early screening of patients and can further limit the spread of disease.
\end{abstract}

Keywords: Dengue fever, NS1 antigen, IgM antibodies, ELISA.

\section{Introduction}

Dengue viruses are Arboviruses capable of infecting humans, and causing diseases. ${ }^{1}$ Dengue virus belongs to the family Flaviviridae, genus Flavivirus. These viruses contain single stranded positive sense RNA. $^{2}$ It has four serotypes DEN-1, DEN-2, DEN-3 and DEN- 4 and a new serotype DEN-5, has been recently identified in the year 2013 in Bangkok. ${ }^{3}$

The Aedes aegypti mosquito is the principal vector that transmits the viruses that cause dengue. The viruses are passed on to humans through the bites of an infective female Aedes mosquito, which acquires the virus while feeding on the blood of an infected individual. Some other diseases like Yellow fever, Zika fever and Chikungunya is also transmitted by these mosquitoes. ${ }^{4}$

It is estimated by W.H.O (World Health Organization) that annual, there is an incidence of 50100 million infections globally; and these number can be as high as 390 million due to inadequate notification, surveillance (both active surveillance as well as passive) and under reporting.

Dengue is endemic in more than 100 countries, in African, Americas, Eastern Mediterranean, South-East Asia and Western Pacific regions. The most severely affected regions are those from the Americas, South-
East Asia and Western Pacific. ${ }^{5}$

According to National Vector Borne Disease Control Programme (NVBDCP) a total of 129166 of dengue cases have been detected with 245 deaths in the year 2016 in India, and in the year 2017 total no. of cases reported was 157220 with 250 deaths reported. ${ }^{6}$

Dengue fever is a severe flu like illness that affects infants, young children and adults, but rarely causes death, Dengue infection causes a wide range of symptoms like high fever $104^{\circ} \mathrm{F}$, severe headache, pain behind the eyes, muscle and joint pains, nausea, vomiting, swollen glands or rash. Symptoms usually last for 2-7 days, after an incubation period of 4-10 days after the bite from an infected mosquito. ${ }^{7,8}$

Severe dengue like dengue hemorrhagic fever (DHF) and dengue shock syndrome (DSS) is a dangerous complication which can lead to plasma leaking, fluid accumulation, respiratory distress, severe bleeding, or organ impairment. Due to its wide range of clinical features, it resembles sign and symptoms of many other diseases. Thus, making it difficult for doctors to diagnose and recognize it timely. There is no specific treatment for dengue/ severe dengue, but early detection and access to proper medical care lowers fatality rates below one percent and helps in early patient management and immediate application of 
appropriate vector control methods which can help to prevent the spread and control of the infection. ${ }^{7,8}$

Laboratory diagnosis procedure for confirming dengue virus infection may include the detection of the viral nucleic acid, virus, antigens or antibodies, or a combination of these techniques. NS1 becomes positive from day one of fever. ${ }^{9}$ Also it usually takes 4-5 and 114 days respectively for anti- DENV IgM and IgG antibodies to become detectable, depending on whether the patient has primary or secondary infection. ${ }^{10}$ ELISA tests based on detection of NS1 antigen and IgM antibodies are commonly used for timely diagnosis. ${ }^{11}$

These tests are too costly, consuming lot of time and commonly unavailable in small laboratories. Lately, commercially available rapid kits detecting the presence of NS1 antigen and/or anti-dengue antibodies have been developed for the rapid detection of dengue. These tests can be done even in distant and far fallen areas with minimum infrastructure and skill. Diagnosis of dengue at an early stage is the only effective way to control disease progression.

We thus tried to determine and evaluate rapid diagnostic tests for NS1 Ag and IgM antibody for acute dengue diagnosis in comparison to ELISA tests in terms of sensitivity and specificity in our hospital.

\section{Aim and Objective}

To compare rapid Immuno-chromatographic test with ELISA for detection of NS1 antigen and IgM antibodies in suspected dengue patients.

\section{Materials and Methods}

A Cross-Sectional study was carried out in the Department of Microbiology, Era's Lucknow Medical College and Hospital from the month of July 2016 to September 2016.

Serum samples of 225 suspected dengue patients were collected, having fever for 5 days or less. All samples were analyzed by Dengue Day 1 Test( J.Mitra \& Co. Pvt. Ltd, India), which detects NS1 antigen, IgM and IgG antibodies, by Panbio Dengue Early ELISA (Standard Diagnostic, INC. Korea) for detecting NS1 antigens and Panbio Dengue IgM Capture ELISA for IgM antibodies.

\section{Results}

In our study, a total of 225 clinically suspected dengue patients were enlisted and their serum samples were tested. Out of total sample tested, predominance of males patients, $65 \%$ (146) were observed, making it a ratio of 1.86: 1 .

Out of all the cases, preponderance were seen in age group of $20-30$ years $(33.33 \%)$ followed by group $10-20$ years $(23.56 \%)$. (Table 1)

Table 1: Age and Gender wise distribution

\begin{tabular}{|c|c|c|c|c|c|c|}
\hline & \multicolumn{4}{|c|}{ Total no. of suspected cases $\mathrm{N}=225$} & & \\
\hline Age Group & \multicolumn{4}{|c|}{ No. of Cases( \%) } & \multirow[b]{2}{*}{ Total } & \multirow[b]{2}{*}{$(\%)$} \\
\hline (Years) & Males & $\%$ & Females & $\%$ & & \\
\hline $0-10$ & 14 & 9.58 & 5 & 6.33 & 19 & 8.44 \\
\hline $10-20$ & 34 & 23.29 & 19 & 24.05 & 53 & 23.56 \\
\hline $20-30$ & 47 & 32.19 & 28 & 35.44 & 75 & 33.33 \\
\hline $30-40$ & 18 & 12.33 & 9 & 11.39 & 27 & 12.0 \\
\hline $40-50$ & 15 & 10.28 & 8 & 10.13 & 23 & 10.22 \\
\hline $50-60$ & 10 & 6.85 & 6 & 7.60 & 16 & 7.11 \\
\hline$>60 \mathrm{yrs}$ & 08 & 5.48 & 4 & 5.06 & 12 & 5.34 \\
\hline Total $(\mathrm{N})$ & 146 & $65 \%$ & 79 & $35 \%$ & 225 & $100 \%$ \\
\hline \multicolumn{3}{|c|}{ Males } & \multicolumn{3}{|c|}{ Females } & Total \\
\hline \multicolumn{3}{|c|}{$146(65 \%)$} & \multicolumn{3}{|c|}{$79(35 \%)$} & $225(100 \%)$ \\
\hline
\end{tabular}

Total number of patients detected positive by either NS1 ELISA or IgM ELISA were 99 (44\%), and it was also observed that majority of the patients were males $69(69.70 \%)$. A total of 6 patients were detected positive by both NS1 as well as IgM ELISA. (Table 2).

Table 2: Sex wise distribution of dengue positive cases by elisa

\begin{tabular}{|l|c|c|}
\hline & Dengue Positive \\
\hline Sex & Number & Percentage \\
\hline Male & 69 & 69.70 \\
\hline Female & 30 & 30.30 \\
\hline Total & 99 & 100 \\
\hline
\end{tabular}

After analyzing the results a total Sensitivity of 92 percent was seen with NS1 antigen, and Specificity of 98 percent, with Positive predictive valve of 95.83 percent, while data accuracy was calculated to be around 96 percent. 
Table 3: Comparison of NS1 ELISA and ICT for NS1 antigen

\begin{tabular}{|l|c|c|c|}
\hline & ELISA +ve & ELISA -ve & TOTAL \\
\hline RAPID +ive & 69 & 3 & 72 \\
\hline RAPID -ive & 6 & 147 & 153 \\
\hline & 75 & 150 & 225 \\
\hline
\end{tabular}

\begin{tabular}{|c|c|}
\hline Sensitivity & $92 \%$ \\
\hline Specificity & $98 \%$ \\
\hline PPV & $95.83 \%$ \\
\hline NPV & $96.08 \%$ \\
\hline DA & $96 \%$ \\
\hline
\end{tabular}

Table 4 depicts a total sensitivity of 90 percent with $\operatorname{IgM}$ and a total Specificity of 100 percent. Positive Predictive Value was found to be 100 percent, while Data Accuracy of 98.67 percent was observed.

Table 4: Comparison of capture ELISA and ICT for IgM

\begin{tabular}{|l|c|c|c|}
\hline & ELISA +ve & ELISA -ve & TOTAL \\
\hline RAPID +ive & 27 & 0 & 27 \\
\hline RAPID -ive & 3 & 195 & 198 \\
\hline & 30 & 195 & 225 \\
\hline
\end{tabular}

\begin{tabular}{|c|c|}
\hline Sensitivity & $90 \%$ \\
\hline Specificity & $100 \%$ \\
\hline PPV & $100 \%$ \\
\hline NPV & $98.5 \%$ \\
\hline DA & $98.67 \%$ \\
\hline
\end{tabular}

\section{Discussion}

It has been observed in tropical countries that most of the febrile diseases have similar signs and symptoms which can often resembles those of dengue, making it difficult to diagnose without laboratory confirmation, thus increasing the disease burden. ${ }^{12}$

In our study, Serum samples of 225 suspected dengue patients were analyzed by Dengue rapid test \& ELISA for detection of NS1 antigen and IgM antibodies. We found that majority of the patients 75 $(33.33 \%)$ were from the age group of 20-30 years. The finding of the present study are consistent with the study conducted by $\mathrm{T}$. Begum et $\mathrm{al}^{13}$ who observed around 43 percent patients included in their study were from the age group of 15-30 years. Almost similar results were also obtained by Gore MM et al, ${ }^{14}$ Raju BJ et $a \mathrm{al},{ }^{15} \mathrm{NeerjaM}$ et $\mathrm{al}^{16}$ and Dash PK et al ${ }^{17}$

We also observed that majority of patients were predominantly males $(65 \%)$ than females $(35 \%)$ i.e. 1.86: 1. Our observations were similar to the study conducted by Raju BJ et al ${ }^{15}$ who also found predominance of males (61\%) over females (39\%). Similar results were also obtained by Dash PK et al ${ }^{17}$ and Neeraja $\mathrm{M}$ et $\mathrm{al}^{16}$ in their studies.

Many studies conducted in various hospital have obtained a wide range of sensitivity (48.5 to $98.7 \%$ ) and specificity (71.42 to $100 \%)$ of ICT based RDTs compared with ELISA, similarly in the our study, we found a sensitivity and specificity of $92 \%$ and $98 \%$ respectively for NS1 antigen detection and $90 \%$ and
$100 \%$ respectively for IgM Antibody detection. In a study conducted by Kaylan D et.al ${ }^{17}$ found high positivity rate in $22.5 \%$ ( 9 out of 40 cases) compared to conventional ELISA that showed 5\% positivity (2 out of 40 cases) in acute dengue cases.

Observations in our study are similar to the studies conducted by various researchers and most of them showed ELISA to have a higher detection power. The diagnostic sensitivity and specificity can be increased by combining the results obtained by NS1 and IgM Assay. Indistinguishably, results of our study were similar to the results obtained by other authors.

The predictive values (positive as well as negative) of the different diagnostic kits will show diverse results depending upon the incidence and prevalence of dengue and other febrile illnesses.

Dengue is rapidly emerging pandemic viral disease extending not only in tropical regions but to all different parts of the world, with the increase in incidence and prevalence of dengue cases and their reporting, use of NS1 antigen detection can help in the early identification of positive cases. Early detection of positive patients can further assist in timely clinical intervention, investigation, and disease prevention as well as its control.

\section{Conclusion}

In our study we found a high sensitivity and specificity of rapid diagnostic tests for early detection of dengue. These kits are highly suitable for early 
detection of positive cases, as with high sensitivity and specificity it can help in early screening of patients and can further limit the spread of disease. These tests are easy to execute, and also need no professionalism or extravagant equipment's and can help in an early detecting of cases, there by accelerating an early diagnosis, especially in centers where facilities for ELISA are not available.

\section{Acknowledgement}

We show our gratitude towards the Department of Pediatrics and Department of Medicine for their assistance and for providing the samples required for our study and also for their comments that greatly helped in execution of the study. We would also like to thank the Department of Community Medicine for their help in carrying out required statistical analysis.

\section{References}

1. Park K. Park's Textbook of Preventive Medicine.24th edition. Jabalpur: m/s Banarsidas Bhanot; 2017. p. 261-262.

2. K Surrender.Textbook of Microbiology. $1^{\text {st }}$ Edition. Jaypee brothers Medical Publisher (p) Ltd 2012. p581-582

3. Sastry AS. Bhat SK. Aroboviruses $1^{\text {st }}$ Edition. Jaypee Essentials of Medical Microbiology First edition. Jaypee Brothers Medical Publishers (P) Ltd: 2016. p 489- 491.

4. CDC.Gov. 2017 [Cited on 20 November 2017]. Available from

https://www.cdc.gov/dengue/epidemiology/index.html

5. W.H.O. Int. Dengue control [ Cited on 20 November 2017] Available from http://www.who.int/denguecontrol/epidemiology/en/

6. NVBDCP (National Vector Borne Disease Control Programme . Directorate General of health services. Minister of health and family welfare ).Available from http://nvbdcp.gov.in/den-cd.html

7. W.H.O. Int Mediacentre Factsheets. [ cited on $10^{\text {th }}$ November 2017] Available from http://www.who.int/mediacentre/factsheets/fs117/en/

8. Parkash O, Hanim Shueb R. Diagnosis of Dengue Infection Using Conventional and Biosensor Based Techniques. Ploss A, ed. Viruses. 2015;7(10):54105427. doi:10.3390/v7102877

9. Sastry AS. Bhat SK. Aroboviruses $1^{\text {st }}$ Edition.JaypeeEssentials of Medical Microbiology First edition.Jaypee Brothers Medical Publishers (P) Ltd: 2016. P. 490- 491

10. Ahmed NH, Broor S. Comparison of NS1 antigen detection ELISA, real time RT- PCR and virus isolation for rapid diagnosis of dengue infection in acute phase. Journal of vector borne diseases. 2014;51(3):194-9. pmid:25253212

11. Andries AC, Duong V, Nagan C, Ong S, Huy R, Sroin $\mathrm{KK}$ et al. Field evaluation and impact on clinical management of a rapid diagnostic kit that detects dengue NS1, IgM and IgG. PLoS Negl Trop Dis. 2012;6(12): e199

12. Capeding MR, Chua MN, Hadinegoro SR, Hussain IIHM, Nallusamy R, Pitisuttithum P, et al. (2013) Dengue and Other Common Causes of Acute Febrile Illness in Asia: An Active Surveillance Study in Children. PLoS
Negl Trop Dis 7(7): e2331.

https://doi.org/10.1371/journal.pntd.0002331

13. Begum, Tabasum, M. N. Sumana, and H. Basavana Gowdappa. "Evaluation of Rapid ICT in comparison with MAC-ELISA in diagnosis of dengue fever at a tertiary care hospital, South India." infection 2013 (2013).

14. Gore MM. Need for constant monitoring of dengue infection. Indian J Med Res 2005;121(1):9-12.

15. Raju BJ, Rajaram G. Prevalence of dengue fever and dengue hemorrhagic fever in government general hospital tirupati . Int J Res Health Sci 2013;1(1):23-27.

16. Neeraja M, Lakshmi V, Teja VD, Umabala P, Subbalakshmi MV. Serodiagnosis of dengue virus infection in patients presenting to a tertiary care hospital. Indian J Med Microbiol 2006;24(4):280-282.

17. Dash PK, Sharma S, Srivastava A, Santhosh SR, Parida MM, Neeraja M, et al. Emergence of dengue virus type 4 (genotype I) in India. Epidemiol Infect 2011;139(6):857-61.

18. Kalyani D, Bai MM. Evaluation of immunochromatographic test in early serological diagnosis of dengue fever. Indian J Pathol Microbiol 2012;55:610-1 